consequence, the height of the unit cell is decreased by about 5 per cent and the width and breadth are both increased by about 2.5 per cent. Such a change of shape - particularly when it is brought about at the lower temperatures in the zone of ordering - introduces very severe internal strains, with a consequent increase in hardness and strength.

This mechanism of atomic rearrangement at low temperatures, first established by studies on the simple binary alloys of gold and copper, has since been found to be a means of modifying the properties of many important industrial materials. Not only is it responsible - at least in part - for the effects of "tempering" the S. S. White dental alloy, but it is also involved in developing the extraordinary magnetic properties of cobalt-platinum hard magnetic alloys, in the high damping properties of the manganese-copper-nickel alloys, in the hardness of $\beta$ brasses, and even in the properties of many steels after heat treatment.

Today, ordering reactions are utilised to harden copper-containing gold alloys used for wipers in sliding electrical contacts. Appropriate heat treatment of a 62.5 per cent gold alloy containing copper and silver will, for example, produce a tensile strength of some 60 to 70 ton $/ \mathrm{in}^{2}$ with a Vickers hardness of about 275 . Ordering reactions are believed to occur in a range of alloys in the binary gold-vanadium, gold-zinc and gold-cadmium systems, though not a great deal is known of the precise mechanisms involved. It seems possible also that ordering may influence the performance of gold-palladium thermocouple alloys as well as of gold-nickel electrical contact alloys. There is undoubtedly scope for further research into these and other alloy systems.

\title{
New Moving Coil Meter Suspensions
}

\section{A HIGH STRENGTH GOLD ALLOY LIGAMENT}

The moving coil meter depends for its operation on the efficiency of its suspension. Hitherto, most of those developed for commercial use have incorporated suspension systems in which hardened steel pivots rotate in jewelled housings with delicate hairsprings to provide the restraining force. Such movements suffer from problems of wear, friction and susceptibility to damage from vibration or mechanical shock.

Some of the most delicate laboratory instruments of the galvanometer type use ligament suspensions in which the inherent twist of the ligament provides the restraining force but these have suffered from the disadvantage that the movements are free-hanging and therefore can be used only one way up.

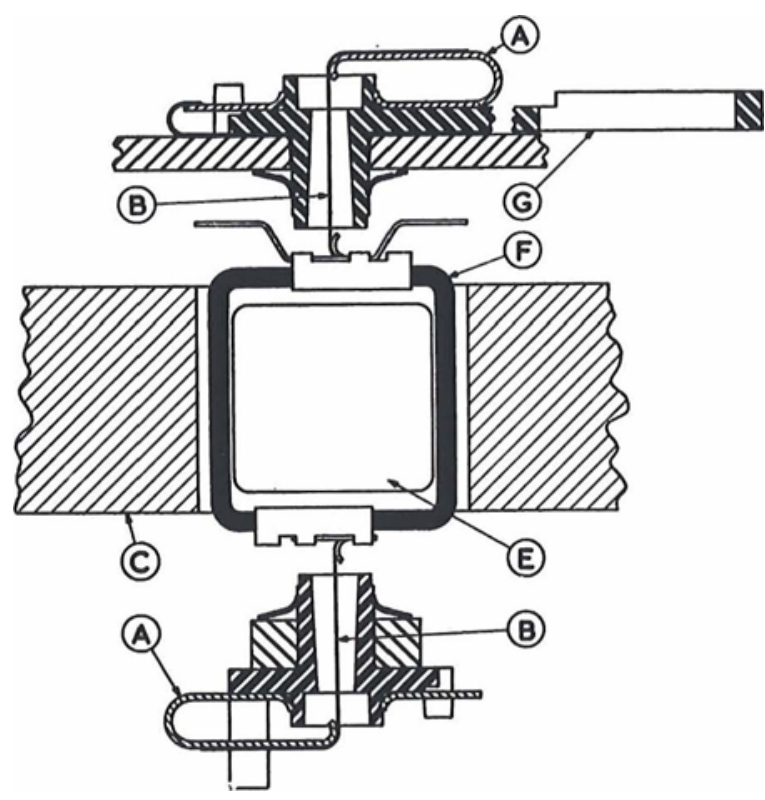

A few years ago Sifam Electrical Instrument Co., Torquay, commenced development of a commercial instrument based on the ligament principle. This operates with two ligaments held taut, one each side of the moving coil, and overcomes previous limitations of ligament suspension while possessing improved sensitivity and robustness.

Sifam refers to these movements as taut-band and they rely upon the ligament giving consistently the desired torque, remaining resilient, and having sufficient tensile strength, together with good electrical characteristics. A special stabilised silver-copper-gold alloy containing 62.5 per cent gold was selected to meet these requirements and it is, moreover, corrosionresistant. The new movement is claimed to be 25 times more sensitive than an equivalent pivoted movement. Hysteresis effects are minimised so that the pointer returns to within 0.25 per cent of true zero even after prolonged operation.

Successful development of these meters is leading Sifam to phase out production of pivoted movement meters altogether. Concentration on the new meter movement has reduced its cost to that of the pivoted movement and expansion of production is foreseen.

The construction of the taut-band movement developed by Sifam for moving coil meters. A - suspension spring, B - gold alloy ligament, C - magnet, $\mathbf{E}$ - core piece, F - coil, G - zero adjustment lever 